\title{
A step forward in our common endeavor
}

\section{Felipe F. Casanueva ${ }^{1}$}

Published online: 16 May 2019

(C) Springer Science+Business Media, LLC, part of Springer Nature 2019

Through the years, Reviews in Endocrine and Metabolic Disorders (REMD) has proven to be a highly useful tool for scientific and clinical endocrinologists. In fact, during the last decade its impact factor was around 5 and currently stands at 4.96, placing it in the first quartile of endocrine journals. Although the impact factor is an undeniable measure of quality, we also need to consider the journal's utility in clinical practice as the word "disorders" in the journal's title directly involves endocrine care. To this end, the high number of downloads, 91,932 in 2018, and the worldwide distribution of Springer journals among learned libraries on each continent further support the journal's utility and efficacy in the field.

However, it is possible to further improve an excellent journal. In striving to become better during this current year, we introduced two important changes. As our readers know, REMD published four "Thematic Issues" annually in the past. As such, each "Thematic Issue" contained around 10 reviews, all focused on a highly relevant topic. The first change is, in addition to thematic reviews, REMD will also publish issues composed of 10-12 individual reviews that are not thematically linked to each other. Such a change was largely requested by our readers and collaborators and comprises the first issue of 2019. This is the beginning of a new experience for REMD and for the current year we expect two thematic Issues, in addition to two issues of individual articles.
The second change introduced is the "presubmission inquiry" procedure. This was implemented to allow potential authors submitting unsolicited reviews to communicate their intentions about the topic of the proposed review, and to allow myself as an Editor-in-Chief to render a preliminary decision. If the proposed review does not fit with the publication strategy for the current year, the author will be notified accordingly, and will be able to submit the paper elsewhere.

Lastly, a high-level endocrine journal depends heavily on the expertise, knowledge, and dedication of its Associate Editors. As such, I would like to thank: Ana Belen Crujeiras (Spain), Maria Fleseriu (USA), Andre Lacroix (Canada), Vera Popovic (Serbia), Pierpaolo Trimboli (Switzerland) and Giovanni Vitale (Italy), for their tremendous work and exceptional commitment to the new REMD orientation. Finally I would also like to thank all the staff and executive members of Springer for their collaboration.

We hope you enjoy this novel "stage" for REMD as we continue to set new standards for this esteemed journal.

Publisher's note Springer Nature remains neutral with regard to jurisdictional claims in published maps and institutional affiliations.
Felipe F. Casanueva

felipe.casanueva@usc.es

1 Santiago de Compostela University, Santiago de Compostela, Spain 